Claremont Colleges

Scholarship@ Claremont

All HMC Faculty Publications and Research

HMC Faculty Scholarship

$1-1-2002$

\title{
Enumeration of Matchings in the Incidence Graphs of Complete and Complete Bipartite Graphs
}

Nicholas Pippenger

Harvey Mudd College

\section{Recommended Citation}

Nicholas Pippenger. "Enumeration of Matchings in the Incidence Graphs of Complete and Complete Bipartite Graphs", SIAM Journal of Discrete Mathematics, 16, 47 (2002).

This Article is brought to you for free and open access by the HMC Faculty Scholarship at Scholarship @ Claremont. It has been accepted for inclusion in All HMC Faculty Publications and Research by an authorized administrator of Scholarship @ Claremont. For more information, please contact scholarship@cuc.claremont.edu. 


\title{
ENUMERATION OF MATCHINGS IN THE INCIDENCE GRAPHS OF COMPLETE AND COMPLETE BIPARTITE GRAPHS*
}

\author{
NICHOLAS PIPPENGER ${ }^{\dagger}$
}

\begin{abstract}
If $G=(V, E)$ is a graph, the incidence graph $I(G)$ is the graph with vertices $V \cup E$ and an edge joining $v \in V$ and $e \in E$ when and only when $v$ is incident with $e$ in $G$. For $G$ equal to $K_{n}$ (the complete graph on $n$ vertices) or $K_{n, n}$ (the complete bipartite graph on $n+n$ vertices), we enumerate the matchings (sets of edges, no two having a vertex in common) in $I(G)$, both exactly (in terms of generating functions) and asymptotically. We also enumerate the equivalence classes of matchings (where two matchings are considered equivalent if there is an automorphism of $G$ that induces an automorphism of $I(G)$ that takes one to the other).
\end{abstract}

Key words. generating functions, asymptotic enumeration

AMS subject classifications. 05A15, 05A16, 05C05

PII. S0895480101395695

1. Introduction. One goal of this paper is the enumeration of matchings in the incidence graphs of certain graphs. There are of course many standard combinatorial results that can be interpreted as counting matchings in a graph. Indeed, for the graphs we consider, the method of inclusion-exclusion yields a summation from which the asymptotic behavior can be obtained by elementary means. We shall also be interested, however, in enumerating equivalence classes of matchings (where two matchings are considered equivalent if there is an automorphism of the underlying graph that induces an automorphism of the incidence graph that takes one matching into the other). For this problem, these standard methods do not serve, and we have had to adopt a different strategy, using Pólya's theory of enumeration [P2, P3] to derive generating functions, and in the bipartite case an analytic method for diagonalizing a bivariate power series introduced by Pippenger [P1]. This new strategy, however, works only for certain highly symmetric graphs. For reasons we will explain later, we are particularly interested in the incidence graphs of complete graphs and of complete bipartite graphs.

We shall denote by $K_{n}$ the complete graph on $n$ vertices, and by $K_{n, m}$ the complete bipartite graph on $n+m$ vertices. If $G=(V, E)$ is a graph, the incidence graph $I(G)$ is the graph with edges $V \cup E$ and an edge joining $v \in V$ and $e \in E$ when and only when $v$ is incident with $e$ in $G$. If $G$ is a graph, we shall denote by $M(G)$ the set of matchings in $G$. (These matchings need not be maximum, or even maximal. Thus $M(G)$ is never empty, since $G$ always has an empty matching. All of the enumerations we present can easily be extended to enumerate matchings by cardinality, simply by inserting an additional indeterminate into the generating functions.)

In section 2 we shall enumerate the matchings $M\left(I\left(K_{n}\right)\right)$ in the incidence graph $I\left(K_{n}\right)$ of the complete graph $K_{n}$. We first do this by inclusion-exclusion, then (as background to what will follow) in a more cumbersome way by Pólya's method.

In section 3 we shall enumerate the equivalence classes $\tilde{M}\left(I\left(K_{n}\right)\right)$ of matchings

\footnotetext{
${ }^{*}$ Received by the editors September 28, 2001; accepted for publication (in revised form) June 20, 2002; published electronically November 6, 2002. This work was supported by an NSERC Research Grant and a Canada Research Chair.

http://www.siam.org/journals/sidma/16-1/39569.html

${ }^{\dagger}$ Department of Computer Science, The University of British Columbia, 2366 Main Mall, Vancouver, BC V6T 1Z4, Canada (nicholas@cs.ubc.ca).
} 
in the incidence graph $I\left(K_{n}\right)$ of the complete graph $K_{n}$. Here, only Pólya's method is applicable. The result is closely related to the enumeration of "functional digraphs" by Harary $[\mathrm{H}]$ and Read $[\mathrm{R}]$.

In section 4 we shall enumerate the matchings $M\left(I\left(K_{n, n}\right)\right)$ in the incidence graph $I\left(K_{n, n}\right)$ of the complete bipartite graph $K_{n, n}$. Again we use both inclusion-exclusion and Pólya's method. To obtain the asymptotic behavior from the generating function, we use the method of Pippenger [P1].

In section 5 we shall enumerate the equivalence classes $\tilde{M}\left(I\left(K_{n, n}\right)\right)$ of matchings in the incidence graph $I\left(K_{n, n}\right)$ of the complete bipartite graph $K_{n, n}$. This result requires combining almost all the techniques introduced in earlier sections.

Most of the methods used in this paper were also used by Pippenger [P1], and many of the calculations done here are along lines similar to ones in that paper. Accordingly, we shall give fewer details for such calculations, referring the reader to that paper when appropriate.

The problems considered in this paper originally arose from the study of "concentrators" for communication switching (see Beneš [B1, B2]). Here, the vertices of $I(G)$ representing edges of $G$ model "clients," while those representing vertices of $G$ model "servers." A "state" of the system, in which some clients are connected in a one-to-one fashion to some servers, then corresponds to a matching in $I(G)$. Enumeration of the matchings thus gives information about the amount of storage required to keep track of the state of the system, while enumeration of the equivalence classes of matchings gives information about the number of essentially different situations that must be considered in formulating a control policy for the system. The 12 elements of $\tilde{M}\left(I\left(K_{4}\right)\right)$ are listed by Beneš [B1, B2].

2. Enumerating $\boldsymbol{M}\left(\boldsymbol{I}\left(\boldsymbol{K}_{\boldsymbol{n}}\right)\right)$. Let $A_{n}$ denote the cardinality of $M\left(I\left(K_{n}\right)\right)$. THEOREM 2.1. We have

$$
A_{n}=\sum_{j \geq 0} \frac{(n)_{2 j}}{2^{j} j !}(-1)^{j} n^{n-2 j}
$$

$\left(\right.$ Here $\left.(n)_{k}=n(n-1) \cdots(n-k+1).\right)$

Proof. Consider a matching $X \in M\left(I\left(K_{n}\right)\right)$. For each edge $\{e, v\} \in X$ (where $e \in E$ is an edge of $K_{n}=(V, E)$ and $v \in V$ is a vertex incident with $e$ ), we shall direct the edge $e=\{v, w\}$ out of $v$ and into $w$. In this way we direct some of the edges of $K_{n}$. These directed edges form the graph of a map $\sigma: D \rightarrow V$ from a subset $D$ of $V$ to $V$. Furthermore, this map does not have any fixed points $(\sigma(v)=v)$ or exchanged pairs of points $(\sigma(v)=w$ and $\sigma(w)=v)$. Conversely, every map $\sigma: D \rightarrow V$ with $D \subseteq V$ having no fixed points or exchanged pairs arises in this way from unique matching in $M\left(I\left(K_{n}\right)\right)$.

The number of maps from a subset of $V$ to $V$ is $(n+1)^{n}$. We can count the number of these having no fixed points or exchanged pairs by using the principle of inclusion-exclusion. There are $n$ possible fixed points, and the fraction of maps having $k$ of them is $\left(\begin{array}{l}n \\ k\end{array}\right)(n+1)^{-k}$. There are $\left(\begin{array}{l}n \\ 2\end{array}\right)$ possible exchanged pairs, and the fraction of maps having $j$ of them is

$$
\frac{1}{j !}\left(\begin{array}{l}
n \\
2
\end{array}\right)\left(\begin{array}{c}
n-2 \\
2
\end{array}\right) \cdots\left(\begin{array}{c}
n-2 j+2 \\
2
\end{array}\right)=\frac{(n)_{2 j}}{2^{j} j !} .
$$

Thus, by inclusion-exclusion, we have 


$$
A_{n}=\sum_{j \geq 0} \sum_{k \geq 0} \frac{(n)_{2 j}}{2^{j} j !}\left(\begin{array}{c}
n-2 j \\
k
\end{array}\right)(-1)^{j+k}(n+1)^{n-2 j-k} .
$$

By the binomial theorem, $\sum_{k \geq 0}\left(\begin{array}{c}n-2 j \\ k\end{array}\right)(-1)^{k}(n+1)^{n-2 j-k}=n^{n-2 j}$. Thus

$$
A_{n}=\sum_{j \geq 0} \frac{(n)_{2 j}}{2^{j} j !}(-1)^{j} n^{n-2 j} .
$$

This last formula can be interpreted by considering vertices of $K_{n}$ unmatched in $X$ to be represented by fixed points, rather than undefined points, of $f$, so that $A_{n}$ is the number of maps from $V$ to $V$ with no exchanged pairs.

Corollary 2.2. As $n \rightarrow \infty$,

$$
A_{n} \sim \frac{n^{n}}{e^{1 / 2}}
$$

Proof. The result of Theorem 2.1 can be rewritten as

$$
A_{n}=n^{n} \sum_{j \geq 0} \frac{(n)_{2 j}}{n^{2 j}} \frac{(-1)^{j}}{2^{j} j !} .
$$

Thus it will suffice to show that

$$
\sum_{j \geq 0} \frac{(n)_{2 j}}{n^{2 j}} \frac{(-1)^{j}}{2^{j} j !} \rightarrow \frac{1}{e^{1 / 2}}
$$

as $n \rightarrow \infty$. Using

$$
\begin{aligned}
\frac{(n)_{2 j}}{n^{2 j}} & =\prod_{0 \leq i<2 j}\left(1-\frac{i}{n}\right) \\
& =\left\{1+O\left(\frac{j^{2}}{n}\right)\right\}
\end{aligned}
$$

for $j \leq \log _{2} n$ and

$$
\left|\frac{(n)_{2 j}}{n^{2 j}} \frac{(-1)^{j}}{2^{j} j !}\right|=O\left(\frac{1}{n^{2}}\right)
$$

for $j>\log _{2} n$, we obtain

$$
\sum_{j \geq 0} \frac{(n)_{2 j}}{n^{2 j}} \frac{(-1)^{j}}{2^{j} j !}=\frac{1}{e^{1 / 2}}\left\{1+O\left(\frac{(\log n)^{2}}{n}\right)\right\}
$$

$\underset{\text { Let }}{\text { since } \sum_{j \geq 0}(-1)^{j} / 2^{j} j !=e^{-1 / 2}}$.

$$
A(z)=\sum_{n \geq 1} \frac{A_{n} z^{n}}{n !}
$$

be the exponential generating function for the sequence $\left\{A_{n}\right\}_{n \geq 1}$. Let $R_{n}$ denote the number of rooted labelled trees on $n$ vertices. Cayley [C] showed that $R_{n}=n^{n-1}$. 
Let

$$
\begin{aligned}
R(z) & =\sum_{n \geq 1} \frac{R_{n} z^{n}}{n !} \\
& =\sum_{n \geq 1} \frac{n^{n-1} z^{n}}{n !}
\end{aligned}
$$

be the exponential generating function for rooted labelled trees. Pólya [P2] and Pólya and Read [P3] showed that $R(z)$ satisfies the functional equation

$$
R(z)=z \exp R(z) .
$$

TheOREM 2.3. We have

$$
A(z)=\frac{\exp \left(-\frac{1}{2} R(z)^{2}\right)}{1-R(z)} .
$$

Proof. Using the interpretation at the end of the proof of Theorem 2.1, we enumerate maps from $V$ (the vertices of $K_{n}$ ) to $V$ having no exchanged pairs. The graph of such a map comprises a number of components. Each component contains a directed cycle, where each vertex of the cycle is the root of a tree in which all edges are directed toward the root. If $R(z)$ is the exponential generating function for labelled rooted trees, then $R(z)^{m} / m$ is the exponential generating function for components containing a cycle of length $m$. Since exchanged pairs correspond to cycles of length 2 , the exponential generating function for components is

$$
\begin{aligned}
C(z) & =\sum_{m \geq 1} \frac{R(z)^{m}}{m}-\frac{1}{2} R(z)^{2} \\
& =\log \frac{1}{1-R(z)}-\frac{1}{2} R(z)^{2} .
\end{aligned}
$$

Applying Pólya's component principle (if the exponential generating function $U(z)$ enumerates labelled components, then the exponential generating function $\exp U(z)$ enumerates labelled structures comprising zero or more components), we obtain

$$
\begin{aligned}
A(z) & =\exp C(z) \\
& =\frac{\exp \left(-\frac{1}{2} R(z)^{2}\right)}{1-R(z)},
\end{aligned}
$$

which completes the proof of the theorem.

We note that Theorem 2.3 can be used to provide an alternative derivation of Corollary 2.2. The singularity of $R(z)$ closest to the origin is at $z=1 / e$, and $R(z)$ has a branch point of order 2 with the expansion

$$
R(z)=1-2^{1 / 2}(1-e z)^{1 / 2}+O(1-e z)
$$

about this point (see Pippenger [P1, p. 96]). Furthermore, we have

$$
|R(z)| \leq \sum_{n \geq 1} \frac{R_{n}|z|^{n}}{n !}<\sum_{n \geq 1} \frac{R_{n} e^{-n}}{n !}=R(1 / e)=1
$$


for $|z|<1 / e$. Thus $A(z)$ also has $z=1 / e$ as its singularity closest to the origin, with the expansion

$$
A(z)=\left(\frac{e}{2}\right)^{1 / 2} \frac{1}{(1-e z)^{1 / 2}}+O(1)
$$

about this point. Applying Darboux's lemma (see Darboux [D] or Knuth and Wilf $[\mathrm{K}]$ ), we obtain

$$
\frac{A_{n}}{n !} \sim\left(\frac{e}{2}\right)^{1 / 2}(-1)^{n}\left(\begin{array}{c}
-\frac{1}{2} \\
n
\end{array}\right) e^{n}
$$

Since $n ! \sim(2 \pi n)^{1 / 2} e^{-n} n^{n}$ and $(-1)^{n}\left(\begin{array}{c}-\frac{1}{2} \\ n\end{array}\right)=\left(\begin{array}{c}2 n \\ n\end{array}\right) / 4^{n} \sim 1 /(\pi n)^{1 / 2}$, we obtain Corollary 2.2 .

3. Enumerating $\tilde{\boldsymbol{M}}\left(\boldsymbol{I}\left(\boldsymbol{K}_{\boldsymbol{n}}\right)\right)$. Let $a_{n}$ denote the cardinality of $\tilde{M}\left(I\left(K_{n}\right)\right)$. Let

$$
a(z)=\sum_{n \geq 1} a_{n} z^{n}
$$

be the ordinary generating function for the sequence $\left\{a_{n}\right\}_{n \geq 1}$. Let $r_{n}$ denote the number of rooted unlabelled trees on $n$ vertices. Let

$$
r(z)=\sum_{n \geq 1} r_{n} z^{n}
$$

be the ordinary generating function for rooted unlabelled trees. Otter $[\mathrm{O}]$ showed that $r(z)$ satisfies the functional equation

$$
r(z)=z \exp \sum_{h \geq 1} \frac{r\left(z^{h}\right)}{h} .
$$

TheOREM 3.1. We have

$$
a(z)=\prod_{m \geq 1} \frac{\exp \left(-\frac{1}{2 m}\left(r\left(z^{m}\right)^{2}+r\left(z^{2 m}\right)\right)\right)}{1-r\left(z^{m}\right)} .
$$

Proof. We proceed as in the proof of Theorem 2.3, with three differences. First, we are enumerating unlabelled, rather than labelled, structures, so we use the ordinary generating function $r(z)$, rather than the exponential generating function $R(z)$, for trees. Second, we use the cycle index $\frac{1}{m} \sum_{i j=m} \phi(j) r\left(z^{j}\right)^{i}$ (where $\phi(j)$ is Euler's function, the number of elements of $\{0,1, \ldots, j-1\}$ relatively prime to $j$ ), rather than $R(z)^{m} / m$, to enumerate unlabelled cycles of length $m$. This gives

$$
\begin{aligned}
c(z) & =\sum_{m \geq 1} \frac{1}{m} \sum_{i j=m} \phi(j) r\left(z^{j}\right)^{i}-\frac{1}{2}\left(r(z)^{2}+r\left(z^{2}\right)\right) \\
& =\sum_{j \geq 1} \frac{\phi(j)}{j} \sum_{i \geq 1} \frac{r\left(z^{j}\right)^{i}}{i}-\frac{1}{2}\left(r(z)^{2}+r\left(z^{2}\right)\right) \\
& =\sum_{j \geq 1} \frac{\phi(j)}{j} \log \frac{1}{1-r\left(z^{j}\right)}-\frac{1}{2}\left(r(z)^{2}+r\left(z^{2}\right)\right)
\end{aligned}
$$


for the ordinary generating function enumerating unlabelled components. Third, we use Pólya's component principle for unlabelled, rather than labelled, structures. (If the ordinary generating function $u(z)$ enumerates unlabelled components, then the ordinary generating function $\exp \sum_{h>1} \frac{1}{h} u\left(z^{h}\right)$ enumerates unlabelled structures comprising zero or more components.) Using $\sum_{j \mid m} \phi(j)=m$ we obtain

$$
\begin{aligned}
a(z) & =\exp \left(\sum_{h \geq 1} \frac{1}{h}\left(\sum_{j \geq 1} \frac{\phi(j)}{j} \log \frac{1}{1-r\left(z^{h j}\right)}\right)-\frac{1}{2}\left(r\left(z^{h}\right)^{2}+r\left(z^{2 h}\right)\right)\right) \\
& =\exp \left(\sum_{m \geq 1} \log \frac{1}{1-r\left(z^{m}\right)}-\frac{1}{2}\left(r\left(z^{m}\right)^{2}+r\left(z^{2 m}\right)\right)\right) \\
& =\prod_{m \geq 1} \frac{\exp \left(-\frac{1}{2 m}\left(r\left(z^{m}\right)^{2}+r\left(z^{2 m}\right)\right)\right)}{1-r\left(z^{m}\right)},
\end{aligned}
$$

which completes the proof of the theorem.

We note that the generating function given in Theorem 3.1 differs merely by the factor of $\prod_{m \geq 1} \exp \left(-\frac{1}{2 m}\left(r\left(z^{m}\right)^{2}+r\left(z^{2 m}\right)\right)\right)$ from the generating function

$$
v(z)=\prod_{m \geq 1} \frac{1}{1-r\left(z^{m}\right)}
$$

derived by Read $[R]$ for the number of unlabelled functional digraphs.

Our next result requires the definition of some constants associated with the generating function $r(z)=\sum_{n \geq 1} r_{n} z^{n}$ for rooted unlabelled trees. We define the function

$$
\begin{aligned}
\Psi(z) & =\sum_{h \geq 2} \frac{r\left(z^{h}\right)}{h} \\
& =\sum_{n \geq 1} r_{n}\left(\log \frac{1}{1-z^{n}}-z^{n}\right) .
\end{aligned}
$$

The singularity of $r(z)$ closest to the origin is at $z=z_{0}$, where $z_{0}$ is the unique positive real solution of the equation $z=\exp -(1+\Psi(z))$. Numerical computation yields $z_{0}=0.3383 \ldots$ We also define the constant $A=1+z_{n} \Psi^{\prime}\left(z_{0}\right)$. Using the expansion

$$
z \Psi(z)=\sum_{n \geq 1} n r_{n}\left(\frac{z^{n}}{1-z^{n}}-z^{n}\right)
$$

numerical computation yields $A=1.215 \ldots$.

Corollary 3.2. As $n \rightarrow \infty$,

$$
a_{n} \sim \frac{c}{n^{1 / 2}}\left(\frac{1}{z_{0}}\right)^{n}
$$

where

$$
c=\frac{\exp \left(-\frac{1}{2} r\left(z_{0}^{2}\right)\right)}{(2 A \pi e)^{1 / 2}} \prod_{h \geq 2} \frac{\exp \left(\frac{1}{2 h}\left(r\left(z_{0}^{h}\right)^{2}+r\left(z_{0}^{2 h}\right)\right)\right)}{1-r\left(z_{0}^{h}\right)}
$$


Proof. The singularity of $r(z)$ closest to the origin is at $z=z_{0}$, and $r(z)$ has a branch point of order 2 with the expansion

$$
r(z)=1-(2 A)^{1 / 2}\left(1-z / z_{0}\right)^{1 / 2}+O\left(1-z / z_{0}\right)
$$

about this point (see Pippenger [P1, p. 104]). Furthermore, we have

$$
|r(z)| \leq \sum_{n \geq 1} r_{n}|z|^{n}<\sum_{n \geq 1} r_{n} z_{0}^{-n}=r\left(z_{0}\right)=1
$$

for $|z|<z_{0}$. Thus $a(z)$ also has $z=z_{0}$ as its singularity closest to the origin, with the expansion

$$
a(z)=\frac{\exp \left(-\frac{1}{2} r\left(z_{0}^{2}\right)\right)}{(2 A e)^{1 / 2}\left(1-z / z_{0}\right)^{1 / 2}} \prod_{h \geq 2} \frac{\exp \left(\frac{1}{2 h}\left(r\left(z_{0}^{h}\right)^{2}+r\left(z_{0}^{2 h}\right)\right)\right)}{1-r\left(z_{0}^{h}\right)}+O(1)
$$

about this point. Applying Darboux's lemma, we obtain

$$
a_{n} \sim \frac{(-1)^{n}}{z_{0}^{n}}\left(\begin{array}{c}
-\frac{1}{2} \\
n
\end{array}\right) \frac{\exp \left(-\frac{1}{2} r\left(z_{0}^{2}\right)\right)}{(2 A e)^{1 / 2}} \prod_{h \geq 2} \frac{\exp \left(\frac{1}{2 h}\left(r\left(z_{0}^{h}\right)^{2}+r\left(z_{0}^{2 h}\right)\right)\right)}{1-r\left(z_{0}^{h}\right)} .
$$

Since $(-1)^{n}\left(\begin{array}{c}-\frac{1}{2} \\ n\end{array}\right) \sim 1 /(\pi n)^{1 / 2}$, we obtain Corollary 3.2 .

The argument used to prove this corollary can also be used to derive the asymptotic behavior of the number $v_{n}$ of unlabelled functional digraphs on $n$ vertices:

$$
v_{n} \sim \frac{1}{(2 A \pi e n)^{1 / 2} z_{0}^{n}} \prod_{h \geq 2} \frac{1}{1-r\left(z_{0}^{h}\right)} .
$$

4. Enumerating $\boldsymbol{M}\left(\boldsymbol{I}\left(\boldsymbol{K}_{\boldsymbol{n}, \boldsymbol{n}}\right)\right)$. Let $B_{n}$ denote the cardinality of $M\left(I\left(K_{n, n}\right)\right)$. THEOREM 4.1. We have

$$
B_{n}=\sum_{j \geq 0}(-1)^{j} j !\left(\begin{array}{l}
n \\
j
\end{array}\right)^{2}(n+1)^{2 n-2 j} .
$$

Proof. Consider a matching $X \in M\left(I\left(K_{n, n}\right)\right.$ ). For each edge $\{e, v\} \in X$ (where $e=\{v, w\} \in E$ is an edge of $K_{n, n}=(V, W, E)$ and $v \in V \cup W$ is a vertex incident with $e$ ), we shall direct the edge $e=\{v, w\}$ out of $v$ and into $w$. In this way we direct some of the edges of $K_{n, n}$. These directed edges form the graph of a map $\sigma: D \rightarrow V \cup W$ from a subset $D$ of the vertices of $V \cup W$ to $V \cup W$. This map exchanges $V$ and $W$. (That is, it takes vertices in $V$ to vertices in $W$, and vertices in $W$ to vertices in $V$.) Furthermore, this map does not have any exchanged pairs of points $(\sigma(v)=w$ and $\sigma(w)=v)$. Conversely, every map $\sigma: D \rightarrow V \cup W$ with $D \subseteq V \cup W$ that exchanges $V$ and $W$ and has no exchanged pairs arises in this way from unique matching in $M\left(I\left(K_{n, n}\right)\right)$.

The number of maps from a subset of $V \cup W$ to $V \cup W$ that take vertices in $V$ to vertices in $W$, and vertices in $W$ to vertices in $V$, is $(n+1)^{2 n}$. We can count the number of these having no exchanged pairs by using the principle of inclusionexclusion. There are $n^{2}$ possible exchanged pairs, and the fraction of maps having $j$ of them is

$$
\frac{j !}{(n+1)^{2 j}}\left(\begin{array}{l}
n \\
j
\end{array}\right)^{2}
$$


Thus, by inclusion-exclusion, we have

$$
B_{n}=\sum_{j \geq 0}(-1)^{j} j !\left(\begin{array}{l}
n \\
j
\end{array}\right)^{2}(n+1)^{2 n-2 j},
$$

which completes the proof of the theorem.

Corollary 4.2. As $n \rightarrow \infty$,

$$
B_{n} \sim e n^{2 n} .
$$

Proof. The result of Theorem 4.1 can be rewritten as

$$
B_{n}=(n+1)^{2 n} \sum_{j \geq 0} \frac{(-1)^{j}}{j !} \frac{(n)_{j}^{2}}{(n+1)^{2 j}} .
$$

As in the proof of Corollary 2.2, we have

$$
\frac{(-1)^{j}}{j !} \frac{(n)_{j}^{2}}{(n+1)^{2 j}}=\frac{(-1)^{j}}{j !}\left\{1+O\left(\frac{j^{2}}{n}\right)\right\}
$$

so that

$$
\sum_{j \geq 0} \frac{(-1)^{j}}{j !} \frac{(n)_{j}^{2}}{(n+1)^{2 j}}=\frac{1}{e}\left\{1+O\left(\frac{(\log n)^{2}}{n}\right)\right\} .
$$

Using $(n+1)^{2 n} \sim e^{2} n^{2 n}$, we obtain the result of the corollary.

Let

$$
B(z)=\sum_{n \geq 1} \frac{B_{n} z^{n}}{n !}
$$

be the exponential generating function for the sequence $\left\{B_{n}\right\}_{n \geq 1}$. Let $B_{n, m}$ denote the cardinality of $M\left(I\left(K_{n, m}\right)\right)$. Let

$$
B(x, y)=\sum_{n, m \geq 1} \frac{B_{n, m} x^{n} y^{m}}{n ! m !}
$$

be the exponential generating function for the sequence $\left\{B_{n, m}\right\}_{n, m \geq 1}$. Our strategy will be to derive the bivariate generating function $B(x, y)$ and then obtain $B(z)$ from it by a method of diagonalization.

Let $R_{n, m}$ denote the number of bicolored rooted labelled trees (that is, the number of rooted labelled trees that, when bicolored, have $n$ vertices with the color of the root and $m$ vertices with the other color). Austin [A] showed that $R_{n, m}=n^{m} m^{n-1}$. Let

$$
\begin{aligned}
R(x, y) & =\sum_{n \geq 1, m \geq 0} \frac{R_{n, m} x^{n} y^{m}}{n ! m !} \\
& =\sum_{n \geq 1, m \geq 0} \frac{n^{m} m^{n-1} x^{n} y^{m}}{n ! m !}
\end{aligned}
$$

be the exponential generating function for the sequence $\left\{R_{n, m}\right\}_{n, m \geq 1}$. Austin $[\mathrm{A}]$ showed that $R(x, y)$ satisfies the functional equation

$$
R(x, y)=x \exp R(y, x) .
$$


Proposition 4.3. We have

$$
B(x, y)=\frac{\exp (R(x, y)+R(y, x)-R(x, y) R(y, x))}{1-R(x, y) R(y, x)} .
$$

Proof. Using the interpretation in the proof of Theorem 4.1, we enumerate maps $\sigma$ from subsets $D \subseteq V \cup W$ to $V \cup W$ that exchange $V$ and $W$ and have no exchanged pairs. The graph of such a map comprises a number of components. Each component either is a rooted tree (where the root is a vertex in $(V \cup W) \backslash D$ at which $\sigma$ is undefined) or contains a directed cycle of even length, where each vertex of the cycle is the root of a tree in which all edges are directed toward the roots. If $R(x, y)$ is the exponential generating function for bicolored rooted labelled trees, then $R(x, y)+R(y, x)$ is the exponential generating function for components that are trees, and $R(x, y)^{m} R(y, x)^{m} / m$ is the exponential generating function for components that contain a cycle of length $2 \mathrm{~m}$. Since exchanged pairs correspond to cycles of length 2 , the exponential generating function for components is

$$
\begin{aligned}
C(x, y) & =\sum_{m \geq 1} \frac{R(x, y)^{m} R(y, x)^{m}}{m}+R(x, y)+R(y, x)-\frac{1}{2} R(x, y) R(y, x) \\
& =\log \frac{1}{1-R(x, y) R(y, x)}+R(x, y)+R(y, x)-\frac{1}{2} R(x, y) R(y, x) .
\end{aligned}
$$

Applying Pólya's component principle (if the exponential generating function $U(x, y)$ enumerates labelled components, then the exponential generating function $\exp U(x, y)$ enumerates labelled structures comprising zero or more components), we obtain

$$
\begin{aligned}
B(x, y) & =\exp C(x, y) \\
& =\frac{\exp (R(x, y)+R(y, x)-R(x, y) R(y, x))}{1-R(x, y) R(y, x)},
\end{aligned}
$$

which completes the proof of the theorem.

THEOREM 4.4. We have

$$
B(z)=\frac{1}{2 \pi} \int_{-\pi / 2}^{3 \pi / 2} \frac{\exp \left(R_{\vartheta}(z)+R_{-\vartheta}(z)-R_{\vartheta}(z) R_{-\vartheta}(z)\right)}{1-R_{\vartheta}(z) R_{-\vartheta}(z)} d \vartheta,
$$

where

$$
R_{\vartheta}(z)=R\left(z e^{i \vartheta}, z e^{-i \vartheta}\right) .
$$

Proof. Each term of the form $x^{n} y^{n}$ in $B(x, y)$ contributes a term of the form $z^{2 n}$ to $B(z)$, whereas each term of the form $x^{n} y^{m}$ (with $n \neq m$ ) in $B(x, y)$ contributes nothing to $B(z)$.

We note that Theorem 4.4 can be used to provide an alternative derivation of Corollary 4.2. Following Pippenger [P1, pp. 97-102], we define

$$
C_{\vartheta}(z)=\frac{R_{\vartheta}(z)+R_{-\vartheta}(z)}{2} .
$$

From the functional equation

$$
R_{\vartheta}(z)=z \exp \left(i \vartheta+R_{-\vartheta}(z)\right)
$$


we have

$$
R_{\vartheta}(z) R_{-\vartheta}(z)=z^{2} \exp \left(2 C_{\vartheta}(z)\right)
$$

This allows the integrand in Theorem 4.4 to be written as

$$
T_{\vartheta}(z)=\frac{\exp \left(2 C_{\vartheta}(z)-z^{2} \exp \left(2 C_{\vartheta}(z)\right)\right)}{1-z^{2} \exp \left(2 C_{\vartheta}(z)\right)} .
$$

As before, the singularities of the integrand are those of $C_{\vartheta}(z)$. There are two such singularities. One of these, at

$$
Z_{\vartheta}^{+}=\exp -\operatorname{cyc} \vartheta,
$$

is closest to the origin when $\vartheta$ is near 0 , and we have the expansion

$$
C_{\vartheta}(z)=\operatorname{cyc} \vartheta-(1+\operatorname{cyc} \vartheta)^{1 / 2}\left(1-z / Z_{\vartheta}^{+}\right)^{1 / 2}+O\left(z-Z_{\vartheta}^{+}\right)
$$

about this point. Here cyc $\vartheta$ denotes a cycloid function having the expansion cyc $\vartheta=$ $1-\vartheta^{2} / 8+O\left(\vartheta^{4}\right)$ for $\vartheta$ near 0 . The other singularity, at

$$
Z_{\vartheta}^{-}=-\exp -\operatorname{cyc}(\vartheta-\pi),
$$

is closest to the origin when $\vartheta$ is near $\pi$, and we have the expansion

$$
C_{\vartheta}(z)=\operatorname{cyc}(\vartheta-\pi)-(1+\operatorname{cyc}(\vartheta-\pi))^{1 / 2}\left(1-z / Z_{\vartheta}^{-}\right)^{1 / 2}+O\left(z-Z_{\vartheta}^{-}\right)
$$

about this point. From Theorem 4.4 we have

$$
\frac{B_{n}}{n !^{2}}=\frac{1}{2 \pi} \int_{-\pi / 2}^{3 \pi / 2}\left[z^{2 n}\right] T_{\vartheta}(z) d \vartheta
$$

We set

$$
\varepsilon(n)=\left(\frac{48 \log n}{n}\right)^{1 / 2}
$$

and break the interval $I=[-\pi / 2,3 \pi / 2)$ into three parts: $J^{+}=[-\varepsilon(n), \varepsilon(n)]$, $J^{-}=[\pi-\varepsilon(n), \pi+\varepsilon(n)]$, and $K=I \backslash\left(J^{+} \cup J^{-}\right)$. For $\vartheta$ in $K$, Cauchy's theorem yields

$$
\left[z^{2 n}\right] T_{\vartheta}(z)=O\left(\frac{e^{2 n}}{n^{3}}\right)
$$

and the integral over $K$ satisfies the same estimate. For $\vartheta$ in $J^{+}$, Darboux's lemma yields

$$
\left[z^{2 n}\right] T_{\vartheta}(z)=\frac{e^{2 n+1}}{4(\pi n)^{1 / 2}}\left\{1+O\left(\frac{(\log n)^{2}}{n}\right)\right\}\left\{1+O\left(\vartheta^{2}\right)\right\} \exp -\left(n \vartheta^{2} / 4\right) .
$$

Thus for the integral over $J^{+}$we have

$$
\frac{1}{2 \pi} \int_{J^{+}}\left[z^{2 n}\right] T_{\vartheta}(z) d \vartheta=\frac{e^{2 n+1}}{4 \pi n}\left\{1+O\left(\frac{(\log n)^{2}}{n}\right)\right\} .
$$

The integral over $J^{-}$satisfies the same estimate, and thus we obtain

$$
\frac{B_{n}}{n !^{2}}=\frac{e^{2 n+1}}{2 \pi n}\left\{1+O\left(\frac{(\log n)^{2}}{n}\right)\right\} .
$$

Since $n ! \sim(2 \pi n)^{1 / 2} e^{-n} n^{n}$, we obtain Corollary 4.2 . 
5. Enumerating $\tilde{\boldsymbol{M}}\left(\boldsymbol{I}\left(\boldsymbol{K}_{\boldsymbol{n}, \boldsymbol{n}}\right)\right)$. Let $b_{n}$ denote the cardinality of $\tilde{M}\left(I\left(K_{n, n}\right)\right)$. Let

$$
b(z)=\sum_{n \geq 1} b_{n} z^{n}
$$

be the ordinary generating function for the sequence $\left\{b_{n}\right\}_{n \geq 1}$. Let $b_{n, m}$ denote the cardinality of $\tilde{M}\left(I\left(K_{n, m}\right)\right)$. Let

$$
b(x, y)=\sum_{n, m \geq 1} b_{n, m} x^{n} y^{m}
$$

be the ordinary generating function for the sequence $\left\{b_{n, m}\right\}_{n, m \geq 1}$. Our strategy will be to derive the bivariate generating function $b(x, y)$ and then obtain $b(z)$ from it by a method of diagonalization.

Let $r_{n, m}$ denote the number of bicolored rooted unlabelled trees (that is, the number of rooted unlabelled trees that, when bicolored, have $n$ vertices with the color of the root and $m$ vertices with the other color). Let

$$
r(x, y)=\sum_{n \geq 1, m \geq 0} r_{n, m} x^{n} y^{m}
$$

be the ordinary generating function for the sequence $\left\{r_{n, m}\right\}_{n, m \geq 1}$. Pippenger [P1] showed that $r(x, y)$ satisfies the functional equation

$$
r(x, y)=x \exp \sum_{h \geq 1} \frac{r\left(y^{h}, x^{h}\right)}{h} .
$$

A positive integer $m$ can be factorized as $m=v(m) \cdot w(m)$, where $v(m)$ is an integral power of 2 and $w(m)$ is an odd integer.

Proposition 5.1. We have

$$
b(x, y)=\frac{f(x, y)+g(x, y)}{2},
$$

where

$$
f(x, y)=\prod_{m \geq 1} \frac{\exp \left(\frac{1}{m}\left(r\left(x^{m}, y^{m}\right)+r\left(y^{m}, x^{m}\right)-r\left(x^{m}, y^{m}\right) r\left(y^{m}, x^{m}\right)\right)\right)}{1-r\left(x^{m}, y^{m}\right) r\left(y^{m}, x^{m}\right)}
$$

and

$$
g(x, y)=\prod_{m \geq 1} \frac{\exp \left(\frac{1}{2 m}\left(r\left(x^{2 m} y^{2 m}\right)-r\left(x^{m} y^{m}\right)^{2}\right)\right)}{1-r\left(x^{m} y^{m}\right)} .
$$

Proof. Using the interpretation in the proof of Theorem 4.1, we enumerate equivalence classes of maps $\sigma$ from subsets $D \subseteq V \cup W$ to $V \cup W$ that exchange $V$ and $W$ and have no exchanged pairs, where now two maps $\sigma$ and $\tau$ are considered equivalent if there is permutation $\pi$ of $V \cup W$ that is (1) either part-preserving (that is, such that $\pi(V)=V$ and $\pi(W)=W$ ) or part-exchanging (that is, such that $\pi(V)=W$ and $\pi(W)=V$ ), and (2) such that $\pi(\tau(v))=\sigma(\pi(v))$ for all $v \in V \cup W$ (which 
means, in particular, that $\tau(v)$ is defined if and only if $\sigma(\pi(v))$ is defined). We shall start by considering only part-preserving permutations. Let $f_{n, m}$ denote the number of equivalence classes of matchings in $I\left(K_{n, m}\right)$ under part-preserving automorphisms of $K_{n, m}$ and $I\left(K_{n, m}\right)$. Let

$$
f(x, y)=\sum_{n, m \geq 1} f_{n, m} x^{n} y^{m}
$$

be the ordinary generating function for the sequence $\left\{f_{n, m}\right\}_{n, m \geq 1}$. We shall show first that $f(x, y)$ is as given in the statement of the theorem.

Next we shall consider part-exchanging permutations. If a matching has no partexchanging automorphism, then it, together with its mate obtained by exchanging $V$ and $W$, are counted twice in $f(x, y)$. If, on the other hand, it has a part-exchanging automorphism (which can happen only when $n=m$ ), then it is counted just once. Let $g_{n, m}$ denote the number of equivalence classes (under part-preserving automorphisms) of matchings in $I\left(K_{n, m}\right)$ that have at least one part-exchanging automorphism. Let

$$
g(x, y)=\sum_{n, m \geq 1} g_{n, m} x^{n} y^{m}
$$

be the ordinary generating function for the sequence $\left\{f_{n, m}\right\}_{n, m \geq 1}$. (We have $g_{n, m}=0$ whenever $n \neq m$, so $g(x, y)$ is actually a power series in the product $x y$.) We shall show that $g(x, y)$ is as given in the statement of the theorem.

Finally, it follows that $b(x, y)=f(x, y) / 2+g(x, y) / 2$, since a matching without a part-exchanging automorphism is counted with weight 1 by the first term, while one with a part-exchanging automorphism is counted with weight $1 / 2$ by the first term, and again with weight $1 / 2$ by the second term.

To derive $f(x, y)$, we proceed as in the proof of Proposition 4.3, with three differences. First, we are enumerating unlabelled, rather than labelled, structures, so we use the ordinary generating function $r(x, y)$, rather than the exponential generating function $R(x, y)$, for trees. Second, we use the cycle index

$$
\frac{1}{m} \sum_{i j=m} \phi(j) r\left(x^{j}, y^{j}\right)^{i} r\left(y^{j}, x^{j}\right)^{i}
$$

rather than $R(x, y)^{m} R(y, x)^{m} / m$, to enumerate unlabelled cycles of length $2 m$. This gives

$$
\begin{aligned}
c(x, y) & =\sum_{m \geq 1} \frac{1}{m} \sum_{i j=m} \phi(j) r\left(x^{j}, y^{j}\right)^{i} r\left(y^{j}, x^{j}\right)^{i}+r(x, y)+r(y, x)-r(x, y) r(y, x) \\
& =\sum_{j \geq 1} \frac{\phi(j)}{j} \sum_{i \geq 1} \frac{r\left(x^{j}, y^{j}\right)^{i} r\left(y^{j}, x^{j}\right)^{i}}{i}+r(x, y)+r(y, x)-r(x, y) r(y, x) \\
& =\sum_{j \geq 1} \frac{\phi(j)}{j} \log \frac{1}{1-r\left(x^{j}, y^{j}\right) r\left(y^{j}, x^{j}\right)}+r(x, y)+r(y, x)-r(x, y) r(y, x)
\end{aligned}
$$

(where we have added the terms $r(x, y)+r(y, x)$ for the components that are trees) for the ordinary generating function enumerating unlabelled components. Third, we use Pólya's component principle for unlabelled, rather than labelled, structures. (If the ordinary generating function $u(x, y)$ enumerates unlabelled components, then the 
ordinary generating function $\exp \sum_{h \geq 1} \frac{1}{h} u\left(x^{h}, y^{h}\right)$ enumerates unlabelled structures comprising zero or more components.) We obtain

$$
\begin{aligned}
f(x, y)= & \exp \sum_{h \geq 1} \frac{1}{h}\left(\sum_{j \geq 1} \frac{\phi(j)}{j} \log \frac{1}{1-r\left(x^{h j}, y^{h j}\right) r\left(y^{h j}, x^{h j}\right)}\right) \\
& \quad+\frac{r\left(x^{h}, y^{h}\right)+r\left(y^{h}, x^{h}\right)-r\left(x^{h}, y^{h}\right) r\left(y^{h}, x^{h}\right)}{h} \\
= & \exp \\
& \left(\sum_{m \geq 1} \log \frac{1}{1-r\left(x^{m}, y^{m}\right)}+\frac{r\left(x^{m}, y^{m}\right)+r\left(y^{m}, x^{m}\right)-r\left(x^{m}, y^{m}\right) r\left(y^{m}, x^{m}\right)}{m}\right) \\
= & \prod_{m \geq 1} \frac{\exp \left(\frac{1}{m}\left(r\left(x^{m}, y^{m}\right)+r\left(y^{m}, x^{m}\right)-r\left(x^{m}, y^{m}\right) r\left(y^{m}, x^{m}\right)\right)\right)}{1-r\left(x^{m}, y^{m}\right) r\left(y^{m}, x^{m}\right)},
\end{aligned}
$$

which completes the derivation of $f(x, y)$.

To derive $g(x, y)$ we proceed as for $f(x, y)$, but we observe that components that do not themselves have a part-exchanging automorphism must come in pairs, along with their mate obtained by exchanging $V$ and $W$. Our goal then is to derive an ordinary generating function for components that have a part-exchanging automorphism. Such a component cannot be a tree, since a tree has its root in one part or the other. Thus it must contain a cycle of even length $2 m$, and its part-exchanging automorphism must rotate this cycle by an odd number of vertices. This odd number of vertices is relatively prime to $v(2 m)$, so the component must comprise $w(m)$ sets of trees, each of which contains $v(m)$ trees along with their $v(m)$ mates. The ordinary generating function for a tree along with its mate is $r(x y, x y)=r(x y)$. Thus the ordinary generating function for such components is

$$
\frac{1}{w(m)} \sum_{i j=w(m)} \phi(j) r\left(x^{j v(m)} y^{j v(m)}\right)^{i} .
$$

Thus the ordinary generating function for all such components (except those associated with exchanged pairs) is

$$
\begin{aligned}
d(x, y) & =\left(\sum_{m \geq 1} \frac{1}{w(m)} \sum_{i j=w(m)} \phi(j) r\left(x^{j v(m)} y^{j v(m)}\right)^{i}\right)-r(x y) \\
& =\left(\sum_{v=2^{t} \geq 1} \sum_{\text {odd } w \geq 1} \frac{1}{w} \sum_{i j=w} \phi(j) r\left(x^{j v} y^{j v}\right)^{i}\right)-r(x y) \\
& =\left(\sum_{v=2^{t} \geq 1} \sum_{\text {odd } j \geq 1} \frac{\phi(j)}{j} \sum_{\text {odd } i \geq 1} \frac{1}{i} r\left(x^{j v} y^{j v}\right)^{i}\right)-r(x y) \\
& =\left(\sum_{v=2^{t} \geq 1} \sum_{\text {odd } j \geq 1} \frac{\phi(j)}{2 j} \log \left(\frac{1+r\left(x^{j v} y^{j v}\right)}{1-r\left(x^{j v} y^{j v}\right)}\right)\right)-r(x y) .
\end{aligned}
$$

The ordinary generating function for components that do not have a part-exchanging automorphism is thus $c(x, y)-d(x, y)$, and for pairs of these components along with 
their mates is $(c(x y, x y)-d(x y, x y)) / 2$. Thus we obtain $g(x, y)$ by applying Pólya's component principle to $(c(x y, x y)-d(x y, x y)) / 2+d(x, y)$. For the first term, we have

$$
\begin{aligned}
\sum_{h \geq 1} \frac{c\left(x^{h} y^{h}, x^{h} y^{h}\right)}{2 h}= & \sum_{h \geq 1} \frac{1}{2 h} \sum_{j \geq 1} \frac{\phi(j)}{j} \log \frac{1}{1-r\left(x^{j h} y^{j h}\right)^{2}} \\
& +\frac{r\left(x^{h} y^{h}\right)}{h}-\frac{r\left(x^{h} y^{h}\right)^{2}}{2 h} \\
= & \sum_{m \geq 1} \frac{1}{2 m} \sum_{j \mid m} \phi(j) \log \frac{1}{1-r\left(x^{m} y^{m}\right)^{2}} \\
& +\frac{r\left(x^{m} y^{m}\right)}{m}-\frac{r\left(x^{m} y^{m}\right)^{2}}{2 m} \\
= & \sum_{m \geq 1} \frac{1}{2} \log \frac{1}{1-r\left(x^{m} y^{m}\right)^{2}} \\
& +\frac{r\left(x^{m} y^{m}\right)}{m}-\frac{r\left(x^{m} y^{m}\right)^{2}}{2 m} .
\end{aligned}
$$

For the last two terms, we have

$$
\begin{aligned}
\sum_{h \geq 1} & \frac{d\left(x^{m}, y^{m}\right)}{h}-\frac{d\left(x^{h} y^{h}, x^{h} y^{h}\right)}{2 h} \\
= & \sum_{h \geq 1} \frac{1}{h} \sum_{u=2^{t} \geq 1} \sum_{\text {odd } j \geq 1} \frac{\phi(j)}{2 j} \log \frac{1+r\left(x^{h u j} y^{h u j}\right)}{1-r\left(x^{h u j} y^{h u j}\right)}-\frac{r\left(x^{h} y^{h}\right)}{h} \\
& -\sum_{h \geq 1} \frac{1}{2 h} \sum_{u=2^{t} \geq 1} \sum_{\text {odd } j \geq 1} \frac{\phi(j)}{2 j} \log \frac{1+r\left(x^{2 h u j} y^{2 h u j}\right)}{1-r\left(x^{2 h u j} y^{2 h u j}\right)}+\frac{r\left(x^{2 h} y^{2 h}\right)}{2 h} \\
= & \sum_{m \geq 1} \frac{1}{m} \sum_{u=2^{t} \geq 1} \sum_{\text {odd } j \mid m} \frac{\phi(j)}{2} \log \frac{1+r\left(x^{m u} y^{m u}\right)}{1-r\left(x^{m u} y^{m u}\right)} \\
& -\sum_{m \geq 1} \frac{1}{2 m} \sum_{u=2^{t} \geq 1} \sum_{m \geq 1} \frac{\phi(j)}{2} \log \frac{1+r\left(x^{2 m u} y^{2 m u}\right)}{1-r\left(x^{2 m u} y^{2 m u}\right)}-\sum_{\text {odd } k \geq 1} \frac{r\left(x^{k} y^{k}\right)}{k} \\
& -\sum_{m \geq 1} \frac{1}{v(v m)} \sum_{u=2^{t} \geq 1} \frac{1}{2} \log \frac{1+r\left(x^{m u} y^{m u}\right)}{1-r\left(x^{m u} y^{m u}\right)} \sum_{\frac{1}{2}} \log \frac{1+r\left(x^{2 m u} y^{2 m u}\right)}{1-r\left(x^{2 m u} y^{2 m u}\right)}-\sum_{\text {odd } k \geq 1} \frac{r\left(x^{k} y^{k}\right)}{k} \\
= & \sum_{\text {odd } k \geq 1} \sum_{u=2^{t} \geq 1} \frac{1}{2} \log \frac{1+r\left(x^{k u} y^{k u}\right)}{1-r\left(x^{k u} y^{k u}\right)} \sum_{\text {odd } k \geq 1} \frac{r\left(x^{k} y^{k}\right)}{k} \\
= & \sum_{m \geq 1} \frac{1}{2} \log \frac{1+r\left(x^{m} y^{m}\right)}{1-r\left(x^{m} y^{m}\right)}-\sum_{\text {odd } k \geq 1} \frac{r\left(x^{k} y^{k}\right)}{k},
\end{aligned}
$$


since $\sum_{\text {odd } j \mid m} \phi(j)=w(m)$. Combining these results, we obtain

$$
\begin{aligned}
g(x, y) & =\exp \sum_{h \geq 1} \frac{c\left(x^{h} y^{h}, x^{h} y^{h}\right)-d\left(x^{h} y^{h}, x^{h} y^{h}\right)+2 d\left(x^{h}, y^{h}\right)}{2 h} \\
& =\prod_{m \geq 1} \frac{\exp \left(\frac{1}{2 m}\left(r\left(x^{2 m} y^{2 m}\right)-r\left(x^{m} y^{m}\right)^{2}\right)\right)}{1-r\left(x^{m} y^{m}\right)}
\end{aligned}
$$

which completes the derivation of $g(x, y)$, and thus the proof of the proposition.

TheOREM 5.2. We have

$$
b(z)=\frac{1}{2 \pi} \int_{-\pi / 2}^{3 \pi / 2} b\left(z e^{i \vartheta}, z e^{-i \vartheta}\right) d \vartheta .
$$

Proof. Each term of the form $x^{n} y^{n}$ in $b(x, y)$ contributes a term of the form $z^{2 n}$ to $b(z)$, whereas each term of the form $x^{n} y^{m}$ (with $n \neq m$ ) in $b(x, y)$ contributes nothing to $b(z)$.

Our next result requires the definition of some constants associated with the generating function $r(x, y)=\sum_{n \geq 1, m \geq 0} r_{n, m} x^{n} y^{m}$ for bicolored rooted unlabelled trees. Define the power series $q(z)=\sum_{n \geq 1} q_{n} z^{n}$ by

$$
q(z)=\sum_{n \geq 1, m \geq 0}(n-m) r_{n, m} z^{n+m}
$$

and then define

$$
B=1-\sum_{n \geq 1} q_{n}\left(\frac{z_{0}^{n}}{1-z_{0}^{n}}-z_{0}^{n}\right) .
$$

Numerical computation yields $B=0.8269 \ldots$. Next define the power series $p(z)=$ $\sum_{n \geq 1} p_{n} z^{n}$ by

$$
p(z)=\sum_{n \geq 1, m \geq 0}(n-m)^{2} r_{n, m} z^{n+m}
$$

and then define

$$
C=-\sum_{n \geq 1} p_{n}\left(\frac{z_{0}^{n}}{\left(1-z_{0}^{n}\right)^{2}}-z_{0}^{n}\right) .
$$

Numerical computation yields $C=-0.4450 \ldots$.

Corollary 5.3. As $n \rightarrow \infty$,

$$
b_{n} \sim \frac{d}{n}\left(\frac{1}{z_{0}}\right)^{2 n},
$$

where

$$
d=\frac{e}{4 \pi\left(B^{2}-4 C\right)^{1 / 2}} \prod_{m \geq 2} \frac{\exp \left(\frac{1}{m}\left(2 r\left(z_{0}^{m}\right)+r\left(z_{0}^{m}\right)^{2}\right)\right)}{1-r\left(z_{0}^{m}\right)^{2}}
$$


Proof. As before, we shall apply Darboux's lemma to the integrand in Theorem 5.2 , and thus we shall be concerned with singularities closest to the origin. Following Pippenger [P1, pp. 104-114], we define

$$
c_{\vartheta}(z)=\frac{c_{\vartheta}(z)+c_{-\vartheta}(z)}{2}
$$

From the functional equation

$$
r_{\vartheta}(z)=z \exp \left(i \vartheta+\sum_{h \geq 1} \frac{r_{-h \vartheta}\left(z^{h}\right)}{h}\right),
$$

we have

$$
r_{\vartheta}(z) r_{-\vartheta}(z)=z^{2} \exp \left(2 \sum_{h \geq 1} \frac{c_{h \vartheta}\left(z^{h}\right)}{h}\right)
$$

As before, the singularities of

$$
t_{\vartheta}(z)=\frac{1}{1-r_{\vartheta}(z) r_{-\vartheta}(z)}
$$

are those of $c_{\vartheta}(z)$. One of these, at

$$
z_{\vartheta}^{+}=z_{0}\left(1+\frac{B^{2}-4 C}{8 A} \vartheta^{2}+O\left(\vartheta^{4}\right)\right),
$$

is closest to the origin when $\vartheta$ is near 0 , and we have the expansion

$$
c_{\vartheta}(z)=\left(1+O\left(\vartheta^{2}\right)\right)-(2 A)^{1 / 2}\left(1+O\left(\vartheta^{2}\right)\right)\left(1-z / z_{\vartheta}^{+}\right)^{1 / 2}+O\left(z-z_{\vartheta}^{+}\right)
$$

about this point. Another singularity, at

$$
z_{\vartheta}^{-}=-z_{0}\left(1+\frac{B^{2}-4 C}{8 A} \vartheta^{2}+O\left(\vartheta^{4}\right)\right),
$$

is closest to the origin when $\vartheta$ is near $\pi$, and we have the expansion

$$
c_{\vartheta}(z)=\left(1+O\left(\vartheta^{2}\right)\right)-(2 A)^{1 / 2}\left(1+O\left(\vartheta^{2}\right)\right)\left(1-z / z_{\vartheta}^{-}\right)^{1 / 2}+O\left(z-z_{\vartheta}^{-}\right)
$$

about this point.

Let us now estimate

$$
t_{n}=\frac{1}{2 \pi} \int_{-\pi / 2}^{3 \pi / 2}\left[z^{2 n}\right] t_{\vartheta}(z) d \vartheta
$$

We set

$$
\varepsilon(n)=\left(\frac{48 \log n}{n}\right)^{1 / 2}
$$

and break the interval $I=[-\pi / 2,3 \pi / 2)$ into three parts: $J^{+}=[-\varepsilon(n), \varepsilon(n)]$, $J^{-}=[\pi-\varepsilon(n), \pi+\varepsilon(n)]$, and $K=I \backslash\left(J^{+} \cup J^{-}\right)$. For $\vartheta$ in $K$, Cauchy's theorem yields

$$
\left[z^{2 n}\right] t_{\vartheta}(z)=O\left(\frac{z_{0}^{-2 n}}{n^{3}}\right)
$$


and the integral over $K$ satisfies the same estimate. For $\vartheta$ in $J^{+}$, Darboux's lemma yields

$$
\left[z^{2 n}\right] t_{\vartheta}(z)=\frac{z_{0}^{-2 n}}{4(A \pi n)^{1 / 2}}\left\{1+O\left(\frac{(\log n)^{2}}{n}\right)\right\}\left\{1+O\left(\vartheta^{2}\right)\right\} \exp -\left(\frac{n \vartheta^{2}\left(B^{2}-4 C\right)}{4 A}\right) .
$$

Thus for the integral over $J^{+}$we have

$$
\frac{1}{2 \pi} \int_{J^{+}}\left[z^{2 n}\right] t_{\vartheta}(z) d \vartheta=\frac{z_{0}^{-2 n}}{4 \pi n\left(B^{2}-4 C\right)^{1 / 2}}\left\{1+O\left(\frac{(\log n)^{2}}{n}\right)\right\} .
$$

The integral over $J^{-}$satisfies the same estimate, and thus we obtain

$$
t_{n}=\frac{z_{0}^{-2 n}}{2 \pi n\left(B^{2}-4 C\right)^{1 / 2}}\left\{1+O\left(\frac{(\log n)^{2}}{n}\right)\right\} .
$$

Let us now estimate

$$
f_{n}=\frac{1}{2 \pi} \int_{-\pi / 2}^{3 \pi / 2} f\left(z e^{i \vartheta}, z e^{-i \vartheta}\right) d \vartheta
$$

Writing $f(x, y)$ as

$$
\begin{aligned}
f(x, y)= & \frac{\exp ((r(x, y)+r(y, x)-r(x, y) r(y, x)))}{1-r(x, y) r(y, x)} \\
& \times \prod_{m \geq 2} \frac{\exp \left(\frac{1}{m}\left(r\left(x^{m}, y^{m}\right)+r\left(y^{m}, x^{m}\right)-r\left(x^{m}, y^{m}\right) r\left(y^{m}, x^{m}\right)\right)\right)}{1-r\left(x^{m}, y^{m}\right) r\left(y^{m}, x^{m}\right)},
\end{aligned}
$$

we see that the asymptotic behavior of $f_{n}$ is determined by that of the denominator of the first factor, which we have already analyzed as $t_{n}$, whereas the numerator of the first factor and all of the remaining factors merely contribute constant factors to the result. Thus we have

$$
\begin{aligned}
f_{n} & \sim e t_{n} \prod_{m \geq 2} \frac{\exp \left(\frac{1}{m}\left(2 r\left(z_{0}^{m}\right)+r\left(z_{0}^{m}\right)^{2}\right)\right)}{1-r\left(z_{0}^{m}\right)^{2}} \\
& \sim \frac{e z_{0}^{-2 n}}{2 \pi n\left(B^{2}-4 C\right)^{1 / 2}} \prod_{m \geq 2} \frac{\exp \left(\frac{1}{m}\left(2 r\left(z_{0}^{m}\right)+r\left(z_{0}^{m}\right)^{2}\right)\right)}{1-r\left(z_{0}^{m}\right)^{2}} .
\end{aligned}
$$

Let us now estimate

$$
g_{n}=\frac{1}{2 \pi} \int_{-\pi / 2}^{3 \pi / 2} g\left(z e^{i \vartheta}, z e^{-i \vartheta}\right) d \vartheta
$$

Every term of $g(x, y)$ is of the form $x^{n} y^{m}$, so that we have $g\left(z e^{i \vartheta}, z e^{-i \vartheta}\right)=g(z, z)$, so that no integration is necessary. Furthermore, $g(z, z)$ has no singularity closer to the origin than $z_{0}^{1 / 2}>z_{0}$, so that we have $g_{n}=O\left(z_{0}^{-n}\right)$, which is negligible as compared with $f_{n}$.

Thus we have

$$
\begin{aligned}
b_{n} & =\frac{f_{n}+g_{n}}{2} \\
& \sim \frac{e z_{0}^{-2 n}}{4 \pi n\left(B^{2}-4 C\right)^{1 / 2}} \prod_{m \geq 2} \frac{\exp \left(\frac{1}{m}\left(2 r\left(z_{0}^{m}\right)+r\left(z_{0}^{m}\right)^{2}\right)\right)}{1-r\left(z_{0}^{m}\right)^{2}},
\end{aligned}
$$

which completes the proof of the theorem. 
Acknowledgment. The author would like to thank Stephane Durocher; his enumeration of $\tilde{M}\left(I\left(K_{5}\right)\right)$ and $\tilde{M}\left(I\left(K_{3,3}\right)\right)$ by hand led to the research reported in this paper.

\section{REFERENCES}

[A] T. L. Austin, The enumeration of point-labelled chromatic graphs and trees, Canad. J. Math., 12 (1960) pp. 535-545.

[B1] V. E. BEnEš, Programming and control problems arising from optimal routing in telephone networks, Bell System Tech. J., 45 (1966) pp. 1373-1438.

[B2] V. E. Beneš, Reduction of network states under symmetries, Bell System Tech. J., 57 (1977) pp. 111-149.

[C] A. CAYley, A theorem on trees, Quart. J. Math., 23 (1889) pp. 376-378.

[D] G. Darboux, Mémoire sur l'approximation des fonctions des très grands nombres, et sur une classe étendue des développements en série, J. Math. Pures Appl., 4 (1878) pp. 5-56, 377-416.

[H] F. Harary, The number of functional digraphs, Math. Ann., 138 (1959) pp. 203-210.

[K] D. E. Knuth And H. S. Wilf, A short proof of Darboux's lemma, Appl. Math. Lett., 2 (1989) pp. 139-140.

[O] R. OtTER, The number of trees, Ann. Math. (2), 49 (1948) pp. 583-599.

[P1] N. PIPPEnger, Enumeration of equicolorable trees, SIAM J. Discrete Math., 14 (2001) pp. 93115.

[P2] G. PÓLYA, Kombinatorische Anzahlbestimmungen für Gruppen, Graphen und chemische Verbindungen, Acta Math., 68 (1937) pp. 145-254.

[P3] G. Pólya and R. C. Read, Combinatorial Enumeration of Groups, Graphs and Chemical Compounds, Springer-Verlag, New York, 1987.

[R] R. C. READ, A note on the number of functional digraphs, Math. Ann., 143 (1961) pp. 109-110. 